\title{
Fine tuning of virulence regulatory pathways in enteric bacteria in response to varying bile and oxygen concentrations in the gastrointestinal tract
}

\author{
Chirantana Sengupta', Sreejana Ray ${ }^{1}$ and Rukhsana Chowdhury ${ }^{1,2^{*}}$
}

\begin{abstract}
After entering the gastrointestinal $(\mathrm{Gl})$ tract on the way to their physiological site of infection, enteric bacteria encounter a remarkable diversity in environmental conditions. There are gross differences in the physico-chemical parameters in different sections of the Gl tract e.g. between the stomach, small intestine and large intestine. Furthermore, even within a certain anatomical site, there are subtle differences in the microenvironment e.g. between the lumen, mucous layer and epithelial surface. Enteric pathogens must not only survive passage through the rapidly changing environments encountered at different niches of the Gl tract but must also appropriately coordinate expression of virulence determinants in response to environmental cues at different stages of infection. There are some common themes in the responses of enteric pathogens to environmental cues, there are also distinct differences that may reflect differences in basic pathogenesis mechanisms. The role of bile and oxygen concentration in spatiotemporal regulation of virulence genes in selected enteric pathogens has been reviewed.
\end{abstract}

Keywords: V. cholerae, Shigella, Salmonella, Virulence, Bile, Oxygen concentration

\section{Introduction}

Enteric and diarrheal diseases remain a major cause of human mortality and morbidity, particularly in developing countries where poor sanitation, lack of safe drinking water and other factors contribute to regular infections specially in children and the aged population. It is estimated that enteric infections are a major cause of childhood mortality in developing countries, killing nearly 1 million children under age 5 every year (WHO report 2013). Repeated episodes of severe diarrhea in the early years of life cause malnutrition and impaired cognitive development which have serious lifelong implications. Some of the most common enteric diseases are caused by bacterial pathogens like Vibrio cholerae, Salmonella enterica, Shigella flexneri, Escherichia coli etc. Development of resistance to multiple antimicrobial drugs among these pathogens is increasing alarmingly with serious consequences on public health (WHO report 2014).

\footnotetext{
* Correspondence: rukhsana@iicb.res.in

'Infectious Diseases and Immunology Division, CSIR-Indian Institute of Chemical Biology, 4 Raja S. C. Mullick Road, Kolkata 700032, India

${ }^{2}$ Academy for Scientific and Innovative Research, CSIR-IICB Campus, Kolkata 700032, India
}

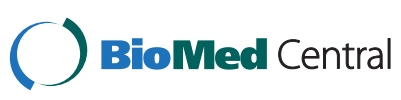

Pathogenic bacteria generally produce an array of virulence factors that allow them to overcome host defenses and survive and multiply in the host body. Disease is often incidental to this primary process. Thus virulence factors include toxins, factors required for adherence to and invasion of host cells, factors conferring resistance to host defenses, those required for modulation of hostile host environments into more favourable ones, and a plethora of accessory factors. Expression of the virulence genes is often tightly regulated by environmental, metabolic and other factors. Many bacterial pathogens have evolved to recognize specific parameters characteristic of the human body as cues for production of virulence factors. The intestinal environment provides a variety of physico-chemical cues to enteric bacteria [1-5]. Bile is a major constituent of the small intestine secreted into the lumen of the duodenum through the bile duct and is thus likely to be encountered by intestinal bacteria in the early stages of infection. Bile is sensed as a host specific cue and regulates expression of virulence factors in certain bacterial pathogens [6]. Also oxygen concentration, presumed to be low in the intestine, is sensed by bacteria and there is some evidence that enteric pathogens express virulence 
factors in response to low oxygen concentrations $[7,8]$. Furthermore, since concentration of bile or oxygen differs in different anatomical sites of the intestine, localized microenvironments may impose spatio-temporal controls to fine tune virulence gene expression at different stages of infection. The virulence regulatory systems of a few enteric bacteria and their fine tuning by bile and oxygen concentration is reviewed.

\section{Virulence regulatory pathways}

Pathogenic bacteria produce an array of virulence factors that allow them to overcome host defenses and survive and multiply in the host body. In $V$. cholerae, the major virulence factors are cholera toxin $(\mathrm{CT})$ and the colonization factor, toxin coregulated pilus (TCP). Expression of the corresponding genes, $c t x A B$ and $t c p A$ is coordinately regulated by transcriptional regulators of the ToxR regulon $[9,10]$. At the top of the hierarchy are AphA, a winged-helix transcription factor, and AphB, a LysR-type regulator, that together activate expression of the $t c p P H$ genes. ТсрP/ТсрH and another transcription regulator ToxR, act synergistically to promote transcription of the toxT gene. ToxT dimers, in turn, activate expression of several virulence genes, including $\operatorname{ctx} A B$ and tcp $A$ (Figure 1A) [11-16]. Although the $V$. cholerae $\operatorname{ctx} A B$ is closely related to the eltAB genes of $E$. coli, the latter is not part of a regulatory cascade and is known to be regulated by cAMP-CRP and the histone-like nucleoid structuring protein $\mathrm{H}-\mathrm{NS}$ under glucose limiting conditions and low temperature respectively $[17,18]$. Both
cAMP-CRP and H-NS also affect $\operatorname{ctx} A B$ expression but by different mechanisms as compared to their effect on elt $A B$ expression. Whereas cAMP-CRP binds to three sites upstream of the E. coli elt $A B$ gene preventing open complex formation and thereby repressing transcription [18], in $V$. cholerae cAMP-CRP represses AphA/AphB-dependent transcriptional activation of the master regulator $t c p P H$ as a result of which, due to a cascading effect, $\operatorname{ctx} A B$ expression is reduced [19]. In $V$. cholerae the global silencer H-NS binds with high affinity to the $\operatorname{ctx} A B$ promoter repressing transcription, the transcription activator ToxT functions as an anti-repressor and is required to overcome the H-NS mediated repression [20]. In contrast, in E. coli, $\mathrm{H}$-NS represses elt $A B$ expression by binding downstream of the transcription start site and aborting transcription elongation at low temperatures, at higher temperatures the repression is partially relieved probably due to changes in DNA curvature that reduce the affinity of H-NS for its binding sites [17]. A similar repression of virulence genes by $\mathrm{H}-\mathrm{NS}$ at low temperatures that is relieved at $37^{\circ} \mathrm{C}$ has also been reported in S. flexneri [21]. Expression of the $S$. flexneri virulence regulon is controlled by a regulatory cascade in which the master regulator VirF activates expression of $\operatorname{vir} B$ and the structural gene icsA. VirB in turn activates transcription of several virulence genes including those coding for invasion proteins and a type III secretion system (T3SS). The transcriptional regulation of virF is complex and is controlled by the global regulators H-NS, Fis, and IHF, as well as the pH-responsive regulator

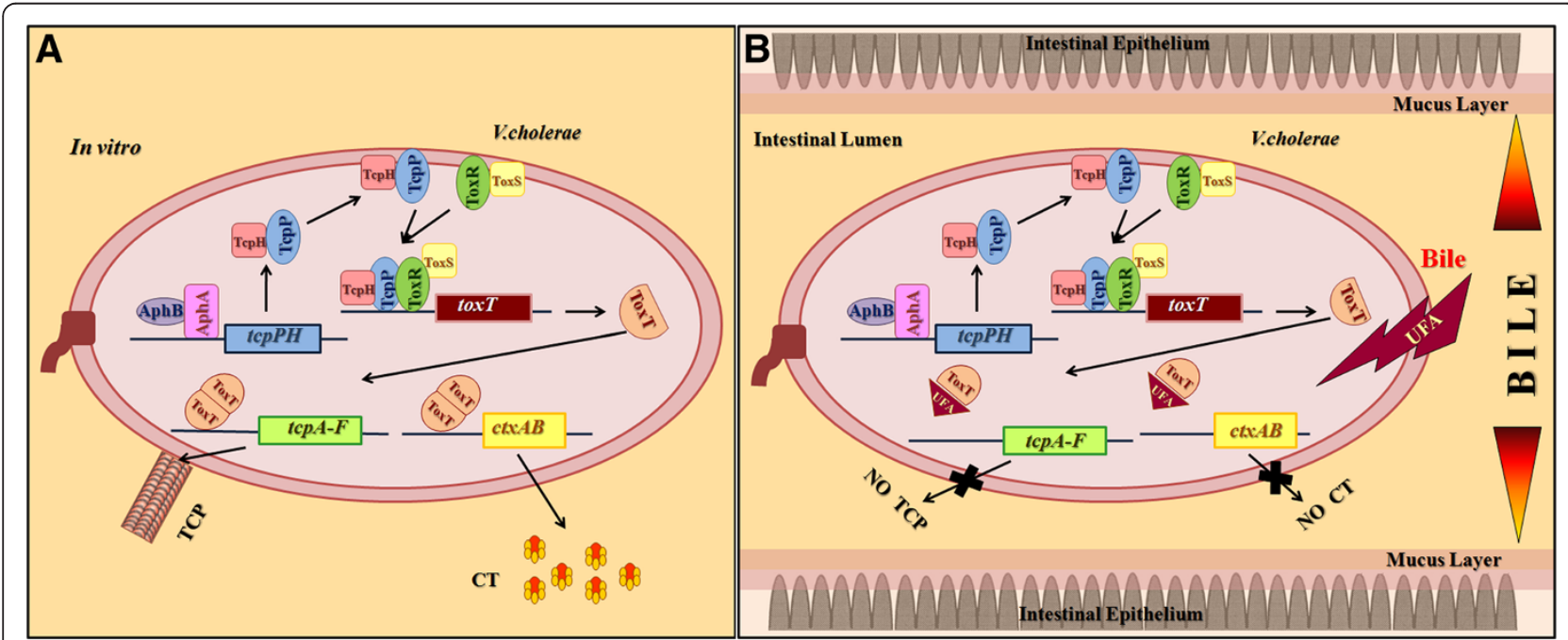

Figure 1 Effect of bile on $\boldsymbol{V}$. cholerae virulence regulation. A. When $V$. cholerae is grown in vitro under permissive conditions without bile the ToxR regulon is activated and the major virulence factors $C T$ and TCP are produced. $\mathbf{B}$. In the intestinal lumen where bile concentration is high, UFA present in bile prevents dimerization of ToxT and thus expression of the virulence genes $\operatorname{ct} A B$ and tcpA is reduced. As the bacteria move away from the lumen through the mucus layer to the underlying epithelium, concentration of bile gradually decreases, consequently $c t \times A B$ and tcpA expression increases. 
CpxR [21-24]. Although mechanistically different, VirB is functionally similar to the $V$. cholerae ToxT since both function as antirepressors to overcome $\mathrm{H}$-NS mediated gene silencing [25].

Two distinct pathogenecity islands SPI-1 and SPI-2 in the Salmonella chromosome encode two distinct virulence associated T3SS, the components of which are expressed in a tightly regulated hierarchical manner at different stages of infection. SPI-1 encoded T3SS is activated by contact with host epithelial cells and translocates effector proteins across the plasma membrane. After the bacteria invade the epithelial cells, the SPI-2 T3SS is expressed within the phagosome and translocates effectors across the vacuolar membrane. At the top of the SPI-1 virulence regulatory cascade are the $\mathrm{AraC}$ family transcription factors, HilC and HilD that together with RtsA, encoded in the core genome, can regulate their own as well as each other's expression through a complex feedback and feedforward mechanism. Each of these regulators can also activate expression of the master regulator HilA that in turn activates expression of the genes encoding the structural components of the SPI-1 T3SS. Unlike SPI-1, SPI-2 encodes a single cognate regulator, the sensor kinase SsrA and the response regulator SsrB [26-28]. Crosstalk between SPI-1 and SPI-2 through HilD induces expression of $\operatorname{ss} A B$ [29]. These core regulatory circuits are modulated by a number of global regulators like H-NS, FIS, OmpR, PhoPQ etc. [30-32].

\section{Recognizing and responding to environmental signals}

A feature of the adaptation strategy of pathogens for optimal survival in their hosts is the recognition of physico-chemical parameters characteristic of the host body and expression of genes related to survival and virulence in response to cues specific to localized microenvironments within the host. This may be achieved either by direct recognition of environmental parameters by dedicated proteins or by integration of virulence gene expression with regulatory circuits that respond to environmental conditions usually involving specific adaptor molecules $(1,2,4,5)$. The regulation of virulence gene expression by bile and oxygen concentration characteristic of the intestinal lumen and their spatiotemporal control will be discussed.

\section{Bile mediated virulence regulation}

Bile is secreted into the lumen of the duodenum from the gall bladder through the bile duct and is inevitably encountered by all enteric bacteria in their human hosts. Bile salts have detergent-like activity [8] that can cause disaggregation of the lipid bilayer structure of cellular membranes, a property that is necessary for protection of the host from invading bacteria. However, Gramnegative enteric bacteria are inherently resistant to bile, partly due to the basic, asymmetric structure of their outer membranes $(\mathrm{OM})$. The protection provided by the $\mathrm{OM}$ is partial and the OM must function in conjunction with energy-dependent efflux systems, which can transport vectorially a diverse array of compounds with little chemical similarity [33]. The best characterized is the AcrAB-TolC efflux pump that can exclude substrates with diverse specificity from the bacteria and thus confers intrinsic resistance to a wide variety of compounds. The AcrAB-TolC efflux pump is required for growth of a number of enteric bacteria including E. coli, V. cholerae and salmonellae, in the presence of bile [34-36]. In addition, salmonellae that has to survive under very high concentrations of bile in the gall bladder employ a number a strategies including peptidoglycan remodeling and changes in outer membrane porins to adapt to high bile concentrations. Furthermore, high level of resistance to bile requires the pleotropic virulence regulator PhoPQ although no individual PhoP-activated (pag) or -repressed ( $p r g)$ gene could be identified that might have a role in bile resistance [37].

Interestingly, many enteric bacteria have further adapted to recognize bile as a signal indicating their entry into the human host, to which they respond by inducing the production of virulence and other factors necessary for survival in the host. Several studies have revealed pathogen specific responses to bile that alter the expression of virulence factors. When $V$. cholerae was grown in the presence of bile, expression of the essential virulence genes $\operatorname{ctx} A B$ and $\operatorname{tcp} A$ was drastically repressed [38]. Since bile is a heterogeneous mixture, crude bile was fractionated, and the components that mediate virulence gene repression were identified. It was shown that the unsaturated fatty acids (UFAs) present in bile, arachidonic, linoleic, and oleic acids were responsible for repression of $\operatorname{ctx} A B$ and tcpA genes [39]. However, expression of the transcriptional activator toxT was not affected and there was no significant difference in ToxT protein levels between $V$. cholerae grown in the presence or absence of bile or UFAs [39]. Subsequently, the crystal structure of ToxT revealed that UFAs can bind to ToxT and keep ToxT in a 'closed' conformation that is not capable of binding DNA [40]. Hence ToxT cannot activate expression of $\operatorname{ctx} A B$ and tcpA in the presence of bile or UFAs and the genes continue to be repressed by H-NS [41]. Bile also causes drastic repression of virulence genes in salmonellae. Salmonellae grown in the presence of bile demonstrated a marked repression of invasion genes and consequently the ability to invade epithelial cells was significantly reduced. Transcription assays demonstrated that bile exerted its effect at the top of the invasion regulatory cascade involving SirA and BarA [42].

A common theme in $V$. cholerae and salmonellae virulence regulation appears to be repression of essential 
virulence factors in the presence of bile. It is paradoxical that bile, that would inevitably be encountered by all enteric bacteria early in infection should repress expression of factors required for virulence in two important and successful enteric pathogens. It is attractive to hypothesize that these pathogens have co-opted bile as an environmental cue to determine their location with respect to different anatomical sites within the intestine so that a spatio-temporal pattern of virulence factor expression could be established. It is known that the concentration of bile gradually decreases from the intestinal lumen to the epithelial surface. In the intestinal lumen where bile concentration is high, $\mathrm{CT}$ and TCP production would be shut off in infecting $V$. cholerae. It might be beneficial for $V$. cholerae to shut off production of CT and TCP in the intestinal lumen for the following reasons. TCP, a pilus if produced in the intestinal lumen might impede progress of the bacteria through the mucus lining of the intestine and retard passage to the underlying epithelial cells. CT production early in infection might lead to profuse fluid secretion from the epithelial cells that could wash away bacteria in the lumen before they could adhere to the epithelial layer. Of greater importance in the early stages of infection would be motility and chemotaxis that would enable the infecting $V$. cholerae to penetrate the mucus layer and adhere to the underlying epithelial cells. Indeed, bile upregulates expression of flagellar genes and motility in $V$. cholerae [38]. Once the bacteria reach the epithelial layer, the lower bile concentration in this microenvironment would allow the repression of virulence genes to be overcome and CT and TCP would be produced (Figure 1B). Similarly, in $S$. Typhimurium, a model has been proposed where bile is recognized as an environmental signal to repress its invasive capacity in the lumen of the intestine, after penetration of the mucous layer and attachment to intestinal epithelial cells, since bile concentration gradually decreases, invasion genes would be expressed and invasion would be initiated. However, although it would be reasonable to assume that bacterial motility would be required to pass through the lumen, and across the mucous membrane to reach the epithelial cells, transcriptome analysis has indicated a bile dependent reduction in expression of motility genes in $S$. Typhimurium, the reason for which is not clear.

The effect of bile on the invasion phenotype of $S$. flexneri is very different from that in the closely related salmonellae. Bile induces production of two outer membrane proteins OspE1/OspE2 that function to promote initial adherence of $S$. flexneri to the intestinal epithelium [43]. Furthermore, when S. flexneri was grown in the presence of deoxycholate (DOC), it was capable of enhanced adherence to and invasion of epithelial cells as compared to bacteria grown without DOC. It has been demonstrated that DOC binds to the invasion protein IpaD at the tip of the external needle of the T3SS to induce recruitment of the translocator protein IpaB into the maturing tip complex thereby facilitating invasion of epithelial cells [44].

\section{Oxygen concentration dependent regulation of virulence}

Oxygen concentration in the intestine is generally considered to be low and anaerobiosis has been shown to regulate virulence of some pathogens. Bacterial adaptation to growth under oxygen deprivation involves several global regulatory systems, like FNR and ArcA/ArcB $[45,46]$. FNR functions as a regulator of virulence genes in several pathogens. FNR positively regulates the expression of several loci involved in flagellar biosynthesis, chemotaxis, acetate metabolism, and SPI-1 invasion genes that have a significant role in Salmonella pathogenesis [47]. FNR also has profound effects on virulence gene expression in S. flexneri, although in $V$. cholerae it has been reported that FNR has no role in virulence regulation $[46,48]$.

Although low oxygen concentration is certainly a characteristic feature of the intestinal lumen, it has been elegantly demonstrated that there is a zone of relative oxygenation adjacent to the GI tract mucosa probably due to diffusion from the capillary network at the tips of the villi. Varying oxygen concentrations at different anatomical sites of the GI tract govern activity of the T3SS of S. flexneri and consequently cell invasion and virulence. Under the microaerobic condition of the intestinal lumen, the anaerobic response regulator FNR is activated and S. flexneri produces extended T3SS needles but secretion of Invasion plasmid antigen (Ipa) effector molecules is reduced in a FNR dependent manner. However, after reaching the relatively aerobic zone adjacent to the mucosal surface, FNR is inactivated and T3SS activation occurs, consequently effectors are secreted (Figure 2). This finely tuned strategy primes the bacteria for invasion while they are in the intestinal lumen but T3SS activation occurs only at its precise site of action, adjacent to the epithelial layer, enhancing invasion and virulence [48].

Spatiotemporal control of virulence gene expression was also observed in the infant mouse model of cholera following $V$. cholerae infection. Using the RIVET technology, it has been demonstrated that expression of the colonization factor TCP encoded by the $t c p A$ gene was induced biphasically in two temporally and spatially separable events, whereas the $\operatorname{ctx} A B$ gene encoding CT was induced monophasically concomitant with the second phase of tcpA induction (Figure 2) [49]. In separate studies it has been demonstrated that under anaerobic conditions $t c p A$ is expressed but $\operatorname{ctx} A B$ is repressed by $\mathrm{H}$-NS. Thus, although expression of $\operatorname{ctx} A B$ and $t c p A$ is 


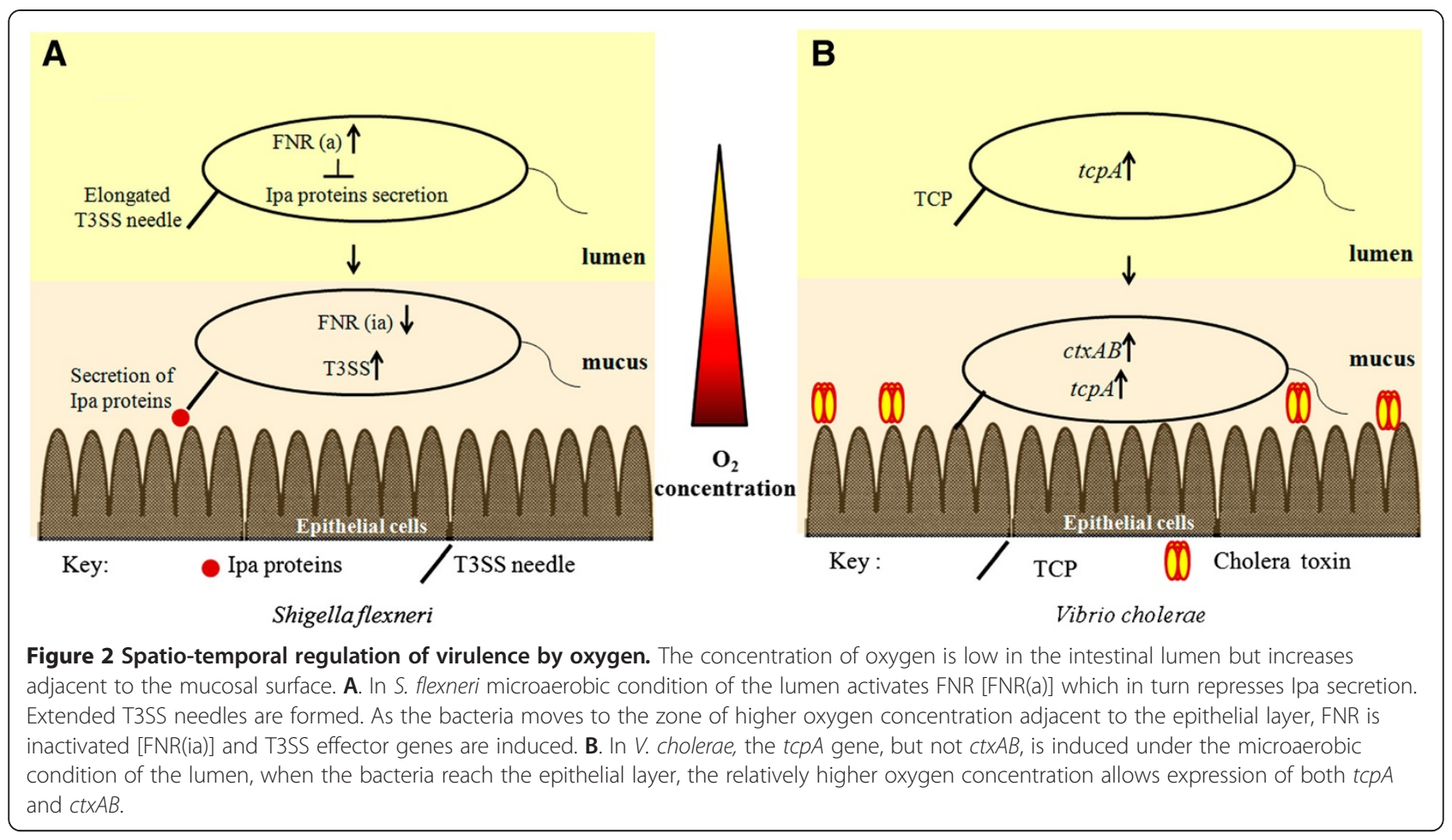

coordinately regulated in vitro, the coordinate regulation is lost in vivo and also under anaerobic conditions [50]. Therefore it is reasonable to hypothesize that the microaerobic condition of the intestinal lumen might allow TcpA production, priming the bacteria for colonization preceding production of $\mathrm{CT}$ that is initiated in a later stage of infection, probably when bacteria reaches the epithelial layer where aeration is relatively high.

\section{Conclusions and future directions}

Many studies have demonstrated that enteric pathogens have evolved to recognize and respond to gross as well as subtle changes in environmental parameters that characterize different niches of the GI tract. The effect of a variety of physico-chemical parameters on expression of virulence genes have been studied in in vitro and in vivo systems, and fine tuning of gene expression at different stages of infection has been established. However, the bacterial sensors of environmental cues are less well characterized. Also few studies have been directed towards identifying changes in the membrane of pathogens at different stages of infection, which could be important in the context of designing vaccines. Finally, although the effect of a variety of physico-chemical parameters and also some metabolites have been studied, the effect of the resident microbiota on gene expression of invading pathogens is not yet understood and could be a focus of future studies.

\section{Competing interests}

The authors declare that they have no competing interests.

\section{Authors' contribution}

CS, SR and RC collected information and drafted the manuscript. RC wrote the final manuscript. All authors read and approved the final manuscript.

\section{Acknowledgement}

We thank all members of the Cholera Group for cooperation, encouragement and helpful discussions during the course of the study. Work in the laboratory was supported by research grant from the CSIR-IICB. C.S. and S.R. would like to thank the Council of Scientific and Industrial Research for research fellowships.

Received: 22 July 2014 Accepted: 1 September 2014 Published: 9 September 2014

\section{References}

1. Kolling G, Wu M, Guerrant RL: Enteric pathogens through life stages. Frontiers Cell Infect Microbiol 2012, 2:114.

2. Mekalanos JJ: Environmental signals controlling expression of virulence determinants in bacteria. J Bacteriol 1992, 174:1-7.

3. Krukonis ES, DiRita VJ: From motility to virulence: Sensing and responding to environmental signals in Vibrio cholerae. Curr Opin Microbiol 2003, 6:186-190.

4. Ferreyra JA, Ng KM, Sonnenburg JL: The Enteric Two-Step: nutritional strategies of bacterial pathogens within the gut. Cell Microbiol 2014, 16:993-1003.

5. Castellani F, Ghidini V, Tafi MC, Boaretti M, Lleo MM: Fate of pathogenic bacteria in microcosms mimicking human body sites. Microb Ecol 2013, 66:224-231.

6. Gunn JS: Mechanisms of bacterial resistance and response to bile. Microbes Infect Institut Pasteur 2000, 2:907-913.

7. Bueno E, Mesa S, Bedmar EJ, Richardson DJ, Delgado MJ: Bacterial adaptation of respiration from oxic to microoxic and anoxic conditions: redox control. Antioxidants Redox Signaling 2012, 16:819-852.

8. Begley M, Gahan CG, Hill C: The interaction between bacteria and bile. FEMS Microbiol Rev 2005, 29:625-651. 
9. Childers BM, Klose KE: Regulation of virulence in Vibrio cholerae: the ToxR regulon. Future Microbiol 2007, 2:335-344.

10. Matson JS, Withey JH, DiRita VJ: Regulatory networks controlling Vibrio cholerae virulence gene expression. Infect Immun 2007, 75:5542-5549.

11. DiRita VJ, Parsot C, Jander G, Mekalanos JJ: Regulatory cascade controls virulence in Vibrio cholerae. Proc Natl Acad Sci U S A 1991, 88:5403-5407.

12. Hase CC, Mekalanos JJ: TcpP protein is a positive regulator of virulence gene expression in Vibrio cholerae. Proc Natl Acad Sci U S A 1998, 95:730-734.

13. Higgins DE, DiRita VJ: Transcriptional control of toxT, a regulatory gene in the ToxR regulon of Vibrio cholerae. Mol Microbiol 1994, 14:17-29.

14. Higgins DE, Nazareno E, DiRita VJ: The virulence gene activator ToxT from Vibrio cholerae is a member of the AraC family of transcriptional activators. J Bacteriol 1992, 174:6974-6980.

15. Kovacikova G, Skorupski K: A Vibrio cholerae LysR homolog, AphB, cooperates with AphA at the tcpPH promoter to activate expression of the ToxR virulence cascade. J Bacteriol 1999, 181:4250-4256.

16. Krukonis ES, Yu RR, Dirita VJ: The Vibrio cholerae ToxR/TcpP/ToxT virulence cascade: distinct roles for two membrane-localized transcriptional activators on a single promoter. Mol Microbiol 2000, 38:67-84.

17. Yang J, Tauschek M, Strugnell R, Robins-Browne RM: The H-NS protein represses transcription of the eltAB operon, which encodes heat-labile enterotoxin in enterotoxigenic Escherichia coli, by binding to regions downstream of the promoter. Microbiology (Reading, England) 2005 151:1199-1208.

18. Bodero MD, Munson GP: Cyclic AMP receptor protein-dependent repression of heat-labile enterotoxin. Infect Immun 2009, 77:791-798.

19. Skorupski K, Taylor RK: Cyclic AMP and its receptor protein negatively regulate the coordinate expression of cholera toxin and toxin-coregulated pilus in Vibrio cholerae. Proc Natl Acad Sci U S A 1997, 94:265-270.

20. Nye MB, Pfau JD, Skorupski K, Taylor RK: Vibrio cholerae H-NS silences virulence gene expression at multiple steps in the ToxR regulatory cascade. J Bacteriol 2000, 182:4295-4303.

21. Porter ME, Dorman CJ: A role for $\mathrm{H}-\mathrm{NS}$ in the thermo-osmotic regulation of virulence gene expression in Shigella flexneri. J Bacteriol 1994 176:4187-4191.

22. Nakayama S, Watanabe $H$ : Identification of $c p x R$ as a positive regulator essential for expression of the Shigella sonnei virF gene. J Bacteriol 1998, 180:3522-3528.

23. Porter ME, Dorman CJ: Positive regulation of Shigella flexneri virulence genes by integration host factor. J Bacteriol 1997, 179:6537-6550

24. Falconi M, Prosseda G, Giangrossi M, Beghetto E, Colonna B: Involvement of FIS in the H-NS-mediated regulation of virF gene of Shigella and enteroinvasive Escherichia coli. Mol Microbiol 2001, 42:439-452.

25. Kane KA, Dorman CJ: Rational design of an artificial genetic switch: Co-option of the H-NS-repressed proU operon by the VirB virulence master regulator. J Bacteriol 2011, 193:5950-5960.

26. Malik-Kale P, Jolly CE, Lathrop S, Winfree S, Luterbach C, Steele-Mortimer O: Salmonella - at home in the host cell. Front Microbiol 2011, 2:125.

27. Cameron $\mathrm{AD}$, Dorman $\mathrm{CJ}$ : A fundamental regulatory mechanism operating through OmpR and DNA topology controls expression of Salmonella pathogenicity islands SPI-1 and SPI-2. PLOS Genet 2012 8:e1002615.

28. Ellermeier CD, Ellermeier JR, Slauch JM: HilD, HilC and RtsA constitute a feed forward loop that controls expression of the SPI1 type three secretion system regulator hilA in Salmonella enterica serovar Typhimurium. Mol Microbiol 2005, 57:691-705.

29. Bustamante VH, Martinez LC, Santana FJ, Knodler LA, Steele-Mortimer O, Puente JL: HilD-mediated transcriptional cross-talk between SPI-1 and SPI-2. Proc Natl Acad Sci U S A 2008, 105:14591-14596.

30. Schechter $L M$, Jain S, Akbar S, Lee CA: The small nucleoid-binding proteins $\mathrm{H}-\mathrm{NS}, \mathrm{HU}$, and Fis affect hilA expression in Salmonella enterica serovar Typhimurium. Infect Immun 2003, 71:5432-5435.

31. Lee AK, Detweiler CS, Falkow S: OmpR regulates the two-component system SsrA-ssrB in Salmonella pathogenicity island 2. J Bacteriol 2000, 182:771-781.

32. Miller SI, Kukral AM, Mekalanos JJ: A two-component regulatory system (phoP phoQ) controls Salmonella Typhimurium virulence. Proc Natl Acad Sci U S A 1989, 86:5054-5058

33. Ma D, Cook DN, Hearst JE, Nikaido H: Efflux pumps and drug resistance in Gram-negative bacteria. Trends Microbiol 1994, 2:489-493.
34. Sylvie Baucheron CM, Praud K, Chaslus-Dancla E, Cloeckaert A: TolC but not AcrB is essential for multidrug-resistant Salmonella enterica serotype Typhimurium colonization of chicks. J Antimicrob Chemother 2005, 55:707-712.

35. Chatterjee A, Chaudhuri S, Saha G, Gupta S, Chowdhury R: Effect of bile on the cell surface permeability barrier and efflux system of Vibrio cholerae. J Bacteriol 2004, 186:6809-6814

36. Ma D, Cook DN, Alberti M, Pon NG, Nikaido $H$, Hearst JE: Genes acrA and $a c r B$ encode a stress-induced efflux system of Escherichia coli. Mol Microbiol 1995, 16:45-55.

37. van Velkinburgh JC, Gunn JS: PhoP-PhoQ-regulated loci are required for enhanced bile resistance in Salmonella spp. Infect Immun 1999, 67:1614-1622.

38. Gupta S, Chowdhury R: Bile affects production of virulence factors and motility of Vibrio cholerae. Infect Immun 1997, 65:1131-1134.

39. Chatterjee A, Dutta PK, Chowdhury R: Effect of fatty acids and cholesterol present in bile on expression of virulence factors and motility of Vibrio cholerae. Infect Immun 2007, 75:1946-1953.

40. Lowden MJ, Skorupski K, Pellegrini M, Chiorazzo MG, Taylor RK, Kull FJ: Structure of Vibrio cholerae ToxT reveals a mechanism for fatty acid regulation of virulence genes. Proc Natl Acad Sci U S A 2009 107:2860-2865.

41. Ghosh A, Paul K, Chowdhury R: Role of the histone-like nucleoid structuring protein in colonization, motility, and bile-dependent repression of virulence gene expression in Vibrio cholerae. Infect Immun 2006, 74:3060-3064

42. Prouty AM, Gunn JS: Salmonella enterica serovar Typhimurium invasion is repressed in the presence of bile. Infect Immun 2000, 68:6763-6769.

43. Faherty CS, Redman JC, Rasko DA, Barry EM, Nataro JP: Shigella flexneri effectors OspE1 and OspE2 mediate induced adherence to the colonic epithelium following bile salts exposure. Mol Microbiol 2012, 85:107-121.

44. Pope LM, Reed KE, Payne SM: Increased protein secretion and adherence to HeLa cells by Shigella spp. following growth in the presence of bile salts. Infect Immun 1995, 63:3642-3648.

45. Compan I, Touati D: Anaerobic activation of $\operatorname{arcA}$ transcription in Escherichia coli: roles of Fnr and ArcA. Mol Microbiol 1994, 11:955-964.

46. Sengupta N, Paul K, Chowdhury R: The global regulator ArcA modulates expression of virulence factors in Vibrio cholerae. Infect Immun 2003, 71:5583-5589.

47. Fink RC, Evans MR, Porwollik S, Vazquez-Torres A, Jones-Carson J, Troxell B, Libby SJ, McClelland M, Hassan HM: FNR is a global regulator of virulence and anaerobic metabolism in Salmonella enterica serovar Typhimurium (ATCC 14028 s). J Bacteriol 2007, 189:2262-2273.

48. Marteyn B, West NP, Browning DF, Cole JA, Shaw JG, Palm F, Mounier J, Prevost MC, Sansonetti P, Tang CM: Modulation of Shigella virulence in response to available oxygen in vivo. Nature 2010, 465:355-358.

49. Lee SH, Hava DL, Waldor MK, Camilli A: Regulation and temporal expression patterns of Vibrio cholerae virulence genes during infection. Cell 1999, 99:625-634.

50. Krishnan HH, Ghosh A, Paul K, Chowdhury R: Effect of anaerobiosis on expression of virulence factors in Vibrio cholerae. Infect Immun 2004, 72:3961-3967

doi:10.1186/s13099-014-0038-9

Cite this article as: Sengupta et al:: Fine tuning of virulence regulatory pathways in enteric bacteria in response to varying bile and oxygen concentrations in the gastrointestinal tract. Gut Pathogens 2014 6:38. 